\title{
Better success rate for root canal therapy when treatment includes obturation short of the apex
}

\author{
What is the optimal obturation length of a root canal to achieve the best \\ prognosis?
}

\section{Schaeffer MA, White RR, Walton RE. Determining the optimal obturation length: a meta-analysis of literature. J Endod 2005; 31:271-274}

Data sources Medline and reference lists of identified articles in English only.

Study selection Initial screening identified studies on humans with radiographic information on the terminal point of obturation. Subsequently studies with (a) a minimum follow-up of at least 2 years; (b) data on termination of obturation/instrumentation; (c) failures of treatment defined adequately (with a minimum radiographic evaluation for failure outlined and defined); (d) data available on success/ failure of root canal therapy in relationship to the obturation/ instrumentation length; and (e) presence or absence of rarefaction included.

Data extraction and synthesis This meta-analysis considered three categories of obturation length from the radiographic apex: (a) 0-1 mm (group A), (b) $>1 \mathrm{~mm}$ but $<3 \mathrm{~mm}$ (group B), (c) obturated past the radiographic apex, including sealer (group $C$ ). The studies were independently assessed for quality with readers blinded to the names of the authors and their institutions, names of the journals, sources of funding, and acknowledgments. Statistical analyses were done using the DerSimonian and Laird estimates.

Results Four studies met the inclusion criteria. In terms of percentage rates of success, the meta-analysis showed that obturation $0-1 \mathrm{~mm}$ short of the apex (group A) was better than obturation 1-3 mm short of the apex (group B); both were superior to obturation beyond the apex (group C). The success rate in group $\mathrm{A}$ was $28.9 \%$ better than group $\mathrm{C}$ and $5.9 \%$ better than group $\mathrm{B}(95 \% \mathrm{Cl}=-3.8 \%, 61.5 \%), P=0.08$ and $(95 \% \mathrm{Cl}=-1.3 \%, 13.1 \%), P=0.11$, respectively. After adjustment for quality, the results remained unchanged.

Conclusions The results demonstrate that obturating materials extruding beyond the radiographic apex correlated with a poorer prognosis. In addition, we realised that standardised protocols are necessary in endodontic procedures and in dental research. If studies are to be compared and the information used clinically, the design of the studies must be similar and repeatable. The results of prospective studies will then be comparable and the data can be combined statistically, thereby creating a more powerful, clinically useful metaanalysis.

\section{Commentary}

It is important to investigate the factors influencing prognosis following root canal therapy. This systematic review focused on the position of termination of root canal filling, among many of the factors potentially influencing the outcome of root canal therapy.

Success rate of obturation beyond the apex was inferior both $0-1 \mathrm{~mm}$ short of the apex and 1 to $3 \mathrm{~mm}$ short of the apex, statistically. However, while obturation $0-1 \mathrm{~mm}$ short of the apex was better than $1-3 \mathrm{~mm}$ short of the apex this was not significant. The overall conclusions provided by the authors are not surprising, as it has been widely accepted that obturating materials are an irritant.

Only four reports were used for the meta-analysis, after 60 papers were initially screened. The inclusion criteria of at least 2 years of follow-up and the performance of radiographic evaluation was a reasonable choice. The authors categorised the obturation length into three groups; 0-1 mm short of the apex, 1-3 mm short of the apex, and obturation beyond the apex. While this seems a pragmatic approach greater discussion of this choice would be helpful. It may also have been useful to raise the limitations of conventional radiographs because of the nature of two-dimensional imaging, and that the root apex is not always located at the apical end of the root.

This study has important implications for clinicians and researchers. From a clinical perspective, obturating materials beyond the radiographic apex during lateral condensation technique clearly decreased the success rate of endodontic treatment. From a research perspective, there is a lack of agreement of definition of success, standardised clinical procedure and evaluation of radiographs. Accumulation of further high quality studies will be required.

\section{Practice point}

- Having root canal obturating materials extruding beyond the radiographic apex decreases the success rates of root canal therapy.

\author{
Toru Naito \\ Department of General Dentistry, Fukuoka Dental College, \\ Fukuoka, Japan
}

Evidence-Based Dentistry (2005) 6, 45.

doi:10.1038/sj.ebd.6400335 